\title{
Comportamento e distribuição de protozoários ciliados (Protista, Ciliophora) no rúmen e no retículo de bovinos submetidos ao jejum*
}

\section{Protozoa ciliate (Protista, Ciliophora) behaviour and distribution in the rumen and reticulum of fasting bovines}

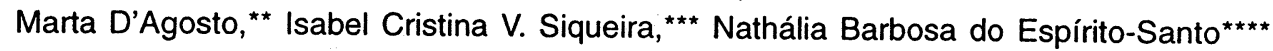

\begin{abstract}
Resumo
Com o objetivo de verificar a distribuição dos ciliados no rúmen e no retículo de bovinos e analisar o possível comportamento de escape de isotriquídeos ao retículo, foram examinadas 18 amostras de conteúdo desses compartimentos, sendo nove de cada. As amostras foram obtidas de bovinos em jejum por, no mínimo, 12 horas, recém-abatidos no Matadouro Municipal de Juiz de Fora, MG, fixadas em formalina a 18,5\% e a quantificação dos ciliados feita com o emprego da câmara Sedgewick-Rafter. Os gêneros dos ciliados observados, sua quantificação e percentuais no rúmen e no retículo, respectivamente, foram os seguintes: Entodinium $\left(10,01 \times 10^{5}, 55,6 \% ; 79,84 \times 10^{4}, 44,4 \%\right)$, Diplodinium $\left(12,32 \times 10^{4}, 54,2 \% ; 10,40 \times 10^{4}\right.$, $45,4 \%)$, Isotricha $\left(14,40 \times 10^{4}, 32 \% ; 30,56 \times 10^{4}, 68 \%\right)$, Dasytricha $\left(25,12 \times 10^{4}, 36,8 \% ; 43,20 \times 10^{4}, 63,2 \%\right)$, Eremoplastron

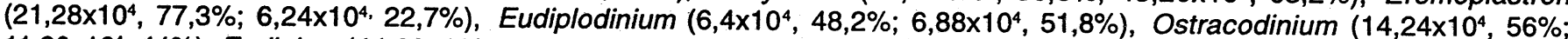
$\left.11,20 \times 10^{4}, 44 \%\right)$, Eodinium $\left(44,80 \times 10^{4}, 58,2 \% ; 32,16 \times 10^{4}, 41,8 \%\right)$, Polyplastron $\left(0,0 ; 0,16 \times 10^{4}, 100 \%\right)$, Diploplastron $\left(2,8 \times 10^{4}\right.$

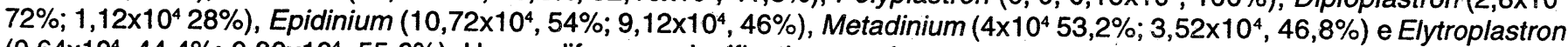
$\left(0,64 \times 10^{4}, 44,4 \% ; 0,80 \times 10^{4}, 55,6 \%\right)$. Houve diferença significativa no número total de ciliados e entre os gêneros Entodinium, Isotricha, Dasytricha, Eremoplastron, Eodinium e Diploplastron ocorrentes no rúmen e no retículo de bovinos nas condições estudadas, destacando-se o maior número total de ciliados no rúmen e o maior número de isotriquídeos no retículo. Os resultados confirmam o escape de isotriquídeos do rúmen ao retículo, verificada pela predominância de 1 sotricha (68\%) e de Dasytricha $(63,2 \%)$ neste compartimento após periodo de jejum, quando foram obtidas as amostras.
\end{abstract}

Palavras-chave: Protozoários ciliados; Isotrichidae; Ophryoscolecidae; rúmen; retículo; bovinos.

\begin{abstract}
Aiming at verifying the distribution of ciliates in the bovine rumen and reticulum and analysing the possible escape behaviour of Isotrichs from the rumen to the reticulum, 18 samples (nine from each cavity) were analysed. The samples were obtained just after death of bovines in the Municipal Slaughter House of Juiz de Fora - MG. They were fixed in 18,5\% formalin and the ciliates quantification was carried out using a Sedgewick-Rafter chamber. The genera of ciliates observed, their quantification and percentage in the rumen and reticulum were, respectively: Entodinium (10,01 x 105, 55,6\%; 79,84 x 104, 44,4\%), Diplodinium $\left(12,32 \times 10^{4}, 54,2 \% ; 10,40 \times 10^{4}, 45,4 \%\right)$, Isotricha $\left(14,40 \times 10^{4}, 32 \% ; 30,56 \times 10^{4}, 68 \%\right)$, Dasytricha $\left(25,12 \times 10^{4}, 36,8 \% ; 43,20\right.$ $\left.\times 10^{4}, 63,2 \%\right)$, Eremoplastron $\left(21,28 \times 10^{4}, 77,3 \% ; 6,24 \times 10^{4}, 22,7 \%\right)$, Eudiplodinium $\left(6,4 \times 10^{4}, 48,2 \% ; 6,88 \times 10^{4}, 51,8 \%\right)$,

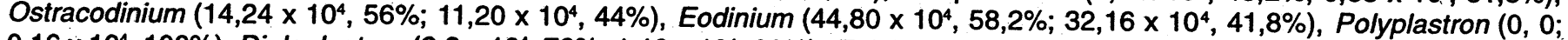
$\left.0,16 \times 10^{4}, 100 \%\right)$, Diploplastron (2,8 $\left.\times 10^{4}, 72 \% ; 1,12 \times 10^{4}, 28 \%\right)$, Epidinium (10,72 $\left.\times 10^{4}, 54 \% ; 9,12 \times 10^{4}, 46 \%\right)$, Metadinium $\left(4 \times 10^{4}, 53,2 \% ; 3,52 \times 10^{4}, 46,8 \%\right)$ and Elytroplastron $\left(0,64 \times 10^{4}, 44,4 \% ; 0,80 \times 10^{4}, 55,6 \%\right)$. There has been significant difference of the total number of ciliates and among the genera Entodinium, Isotricha, Dasytricha, Eremoplastron, Eodinium and Diploplastron found in the rumen and reticulum of bovines, with the occurrence of a greater total number of ciliates in the rumen and a greater number of Isotrichs in the reticulum. The results confirm the escape of Isotrichs from the rumen to the reticulum, verified by the predominance of Isotrichia $(68 \%)$ and Dasytrichia $(63,2 \%)$ in this cavity after a period of fasting, when the samples were collected.
\end{abstract}

Keywords: Protozoa ciliate; Isotrichidae; Ophryoscolecidae; rumen; reticulum; bovines.

\footnotetext{
* Apoio Financeiro: FAPEMIG (Fundação de Amparo à Pesquisa do Estado de Minas Gerais).

** Professora Adjunto, Departamento de Zoologia, Universidade Federal de Juiz de Fora. CEP 36036-330 - Juiz de Fora, MG. dagosto@icb.ufjf.br *** Bolsista de Iniciação Científića. BIC/UFJF.

**** Bolsista de Iniciação Científica. PROBIC/FAPEMIG/UFJF.
} 


\section{Introdução}

O registro de ciliados de rúmen por Gruby e Delafond (1843) deu início a uma série de estudos para melhor conhecimento desses organismos, dentre os quais destacam-se os que abordam sua importância e o papel que desempenham na fisiologia dos ruminantes. A expressão generalizada ciliados de rúmen engloba organismos de grupos diferentes que constituem diversas populações da comunidade ruminal. Apesar das controvérsias quanto à importância dos ciliados aos ruminantes, há concordância quanto a um aspecto: eles não são essenciais ao crescimento e desenvolvimento dos seus hospedeiros.

Diversos fatores, como o tipo e a constituição da dieta, potencial hidrogênio-iônico $(\mathrm{pH})$ do rúmen, intervalo após a alimentação e a migração e escape do rúmen, influem na ocorrência e concentração destes ciliados.

O comportamento de escape dos isotriquídeos ao retículo e sua retenção no rúmen-retículo são potencialmente prejudiciais aos seus hospedeiros, podendo resultar em maior competição por substrato e ingestão de bactérias, ocasionando decréscimo da biomassa bacteriana e dos fluxos de proteína e de energia (LENG et al., 1981).

O presente trabalho objetivou investigar o comportamento de escape de ciliados isotriquídeos do rúmen ao retículo, mediante a análise da distribuição comparativa dos ciliados encontrados no rúmen e no retículo de bovinos submetidos ao jejum.

\section{Material e métodos}

Foram obtidas 18 amostras de conteúdo do rúmen e do retículo em bovinos recém-abatidos no Matadouro Municipal de Juiz de Fora, MG, em agosto de 1997. Os bovinos foram abatidos após jejum de, no mínimo, 12 horas.

As amostras foram fixadas e conservadas segundo Dehority (1984). Para a identificação e quantificação dos gêneros dos ciliados seguiu-se o proposto por Dehority (1984) modificado por D'Agosto e Carneiro (1999).

Os resultados obtidos foram analisados estatisticamente pelo teste Qui-quadrado de Pearson a $5 \%$ de significância.

\section{Resultados e discussão}

Nas amostras de conteúdo do rúmen e do retículo examinadas foram registrados ciliados dos seguintes gêneros: Entodinium, Diplodinium, Isotricha, Dasytricha, Eremoplastron, Diplodinium, Ostracodinium, Eodinium, Polyplastron, Diploplastron, Epidinium, Metadinium e Elytroplastron, não se observando Polyplastron no rúmen.

$\mathrm{Na}$ Tabela 1 estão apresentados os gêneros dos ciliados com os respectivos números médios por mililitro de conteúdo do rúmen e do retículo e percentuais dos gêneros em relação ao número total de ciliados em cada compartimento.

A análise estatística indicou diferença significativa entre a quantificação observada no rúmen e no retículo quanto ao número total de ciliados e entre os seguintes gêneros: Entodinium, Isotricha, Dasytricha, Eremoplastron, Eodinium e Diploplastron, destacando-se o maior número total de ciliados no rúmen e maior número de isotriquídeos no retículo (Tabela 1, Figuras 1 e 2).
Tabela 1: Número médio de ciliados por mililitro de conteúdo do rúmen e do retículo de bovinos recém-abatidos no Matadouro Municipal de Juiz de de Fora, MG, em 1997

\begin{tabular}{|c|c|c|c|c|}
\hline \multicolumn{5}{|c|}{ CILIADOS } \\
\hline Gêneros & $\left(\times 10^{4}\right)^{R}$ & $(\%)$ & $\left(\times 10^{4}\right)$ & $\begin{array}{r}\text { Retículo } \\
(\%) \\
\end{array}$ \\
\hline Entodinium & $100^{a}$ & 55,6 & $79,84^{b}$ & 44,4 \\
\hline Diplodinium & $12,32^{a}$ & 54,2 & $10,40^{a}$ & 45,4 \\
\hline Isotricha & $14,40^{a}$ & 32,0 & $30,56^{b}$ & 68,0 \\
\hline Dasytricha & $25,12^{a}$ & 54,2 & $43,20^{b}$ & 63,2 \\
\hline Eremoplastron & $21,28^{a}$ & 77,3 & $6,24^{b}$ & 22,7 \\
\hline Eudiplodinium & $6,40^{a}$ & 48,2 & $6,88^{a}$ & 51,8 \\
\hline Ostracodinium & $14,24^{a}$ & 56,0 & $11,20^{a}$ & 44,0 \\
\hline Eodinium & $44,80^{a}$ & 58,2 & $32,16^{b}$ & 41,8 \\
\hline Polyplastron & $0^{a}$ & 0 & $0,16^{a}$ & 100,0 \\
\hline Diploplastron & $2,80^{a}$ & 72,0 & $1,12^{b}$ & 28,0 \\
\hline Epidinium & $10,72^{a}$ & 54,0 & $9,12^{a}$ & 46,0 \\
\hline Metadinium & $4,00^{a}$ & 53,2 & $3,52^{a}$ & 46,8 \\
\hline Elytroplastron & $0,60^{a}$ & 44,4 & $0,80^{a}$ & 55,6 \\
\hline TOTAL & $256,96^{a}$ & 52,2 & $235,20^{6}$ & 47,8 \\
\hline
\end{tabular}
significativamente $(P>5 \%)$.

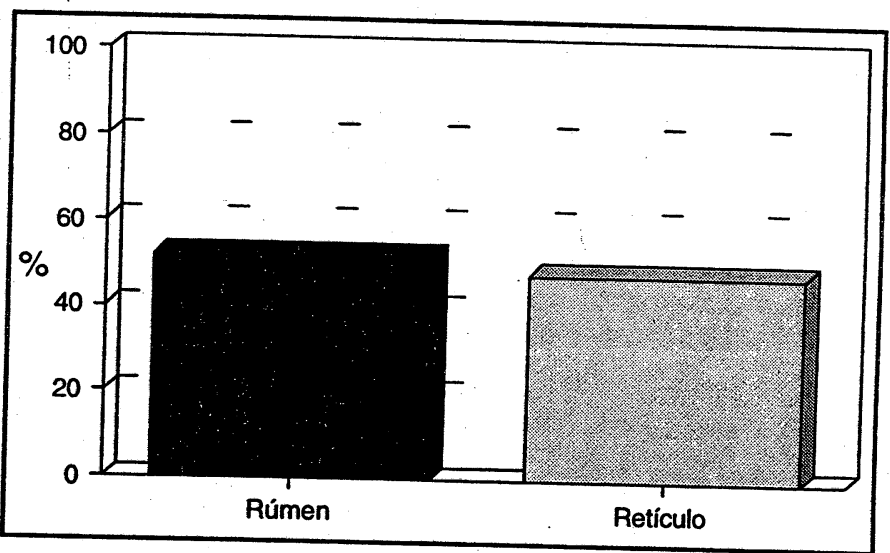

Figura 1: Percentual de ciliados no rúmen e no retículo de bovinos recém-abatidos no Matadouro Municipal de Juiz de Fora, MG, em 1997.

Os isotriquídeos dos gêneros Isotricha e Dasytricha foram os únicos a apresentar maiores populações no retículo do que no rúmen, o que se atribuiu ao escape àquele compartimento relacionado ao período de jejum a que estavam submetidos os bovinos no momento do abate e obtenção das
amostras.

Estes resultados coincidem com os de Abe et al. (1981), que, ao observarem em novilhos abatidos após uma noite sem alimentação uma camada fina de ciliados na parede do 


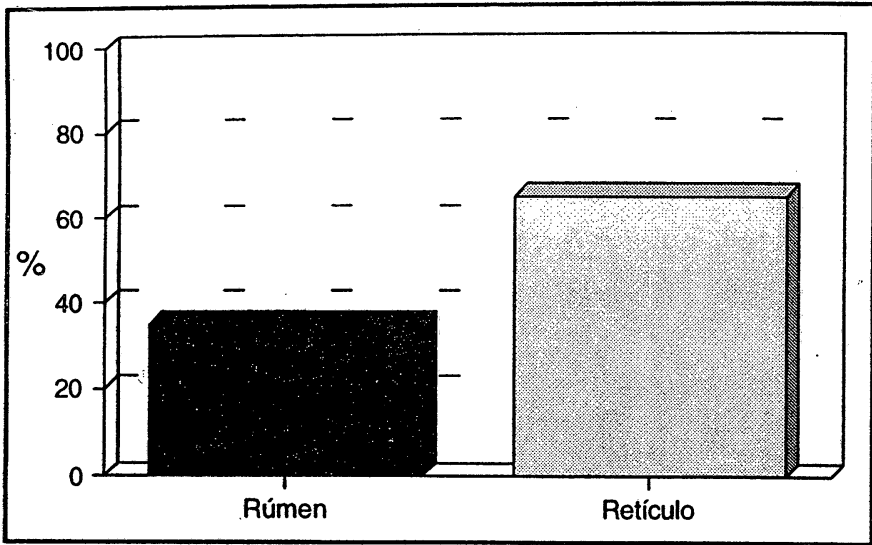

Figura 2: Percentual de Isotrichidae (Isotricha e Dasytricha) no rúmen e no retículo de bovinos recém-abatidos no Matadouro Municipal de Juiz de Fora, MG, em 1997.

\section{Referências bibliográficas}

ABE, M., IRIKI, T., TOBE, N. \& SHIBUI, H. Sequestration of holotrich protozoa in the reticulo-rumen of cattle. Appl. Environ. Microbiol., v. 41, p.758-765, 1981.

D'AGOSTO, M., CARNEIRO, M.E. Evaluation of lugol solution used for counting rumen ciliates. Rev. bras. Zool. v. 16, p. 725729, 1999.

DEHORITY, B.A. Evaluation of subsampling and fixation procedures used for counting rumen Protozoa. Appl. Environ. Microbiol., v. 48, p. 182-185, 1984.

GRUBY, D., DELAFOND, O. Recherches sur des animalcules se développant en grand nombre dans l'estomac et dans les retículo, constituída basicamente de isotriquídeos, o relataram como sinal do comportamento de escape desses ciliados ao retículo. Esses autores destacaram ainda que os isotriquídeos migram ao rúmen após a alimentação dos hospedeiros e escapam para o retículo horas depois. Desta forma, segundo Warner (1962), apesar da biomassa dos isotriquídeos poder representar mais da metade do total da biomassa microbiana no rúmen, é baixa a sua taxa de passagem ao duodeno, pela retenção decorrente do comportamento de escape (Leng et al., 1986).

\section{Conclusão}

Nas condições estudadas, os resultados confirmam indicios do comportamento de escape de isotriquídeos do rúmen ao retículo, verificada pela predominância de Isotricha e de Dasytricha neste compartimento após período de jejum, quando foram obtidas as amostras.

intestins, pendant la digestion des animaux herbivores et carnivores. Compt. Rend. Acad. Sci.,v. 17, p. 1304-1308, 1843.

LENG, R.A., DELLOW, D. \& WARHORN, G. Dynamics of large ciliate protozoa in the rumen of cattle fed on diets of freshly cut grass. Br. J. Nutr., v. 56, p. 455-462, 1986.

LENG, R.A., GILL, M., KEMPTON, T.J., ROWE, J.B., NOLAN, J.V., STACHIW, S.J., PRESTON, T.R. Kinetics of large ciliate protozoa in the rumen of cattle given sugar cane diets. Br. J. Nutr., v.46, p. 371-384, 1981.

OGIMOTO, K., IMAI, S. Atlas of Rumen Microbiology. Tokyo: Japan Scientific Societies Press, viii + 231 p., 1981.

WARNER, A.C.I. Some factors influencing the rumen microbial population. J. Gen. Microbiol., v. 28, p.129-146, 1962. 